\title{
Astro-comb: revolutionizing precision spectroscopy in astrophysics
}

\author{
Claire E. Cramer ${ }^{1,2}$, Chih-Hao Li ${ }^{2}$, Andrew J. Benedick ${ }^{3}$, Alexander \\ G. Glenday ${ }^{1,2}$, Franz X. Kärtner ${ }^{3}$, David F. Phillips ${ }^{2}$, Dimitar \\ Sasselov $^{2,4}$, Andrew Szentgyorgyi ${ }^{2}$, and Ronald L. Walsworth ${ }^{1,2}$ \\ ${ }^{1}$ Dept. of Physics, Harvard University, \\ Cambridge, MA 02138, USA \\ email: ccramer@fas . harvard.edu \\ ${ }^{2}$ Harvard-Smithsonian Center for Astrophysics, Cambridge, MA 02138 USA \\ ${ }^{3}$ Dept. of Electrical Engineering, Computer Science and Research Lab for Electronics, MIT, \\ Cambridge, MA 02138 USA \\ ${ }^{4}$ Dept. of Astronomy, Harvard University, Cambridge, MA 02138, USA
}

\begin{abstract}
Searches for extrasolar planets using the periodic Doppler shift of stellar spectral lines have recently achieved a precision better than $60 \mathrm{~cm} / \mathrm{s}$. To find a 1-Earth mass planet in an Earth-like orbit, a precision of $5 \mathrm{~cm} / \mathrm{s}$ is necessary. The combination of a laser frequency comb with a Fabry-Perot filtering cavity has been suggested as a promising approach to achieve such Doppler shift resolution via improved spectrograph wavelength calibration. Here we report the fabrication of such a filtered laser comb with up to $40 \mathrm{GHz}$ ( 1 Angstrom) line spacing, generated from a $1 \mathrm{GHz}$ repetition-rate source, without compromising long-term stability, reproducibility or spectral resolution. This wide-line-spacing comb (astro-comb) is well matched to the resolving power of high-resolution astrophysical spectrographs. The astrocomb should allow a precision as high as $1 \mathrm{~cm} / \mathrm{s}$ in astronomical readial velocity measurements.
\end{abstract}

\section{Introduction}

The two primary techniques for exoplanet detection - transits and radial velocities provide complementary information about the planets they detect. These techniques have enjoyed wide success, and the goal of detecting Earth-like planets around sun-like stars seems almost within reach. However, to attain this goal, transit searches must be sensitive at the $10^{-5}$ level, and radial velocity searches at the $\mathrm{cm} / \mathrm{s}$ level. This requires an order-ofmagnitude increase in radial velocity sensitivity. While the best reported radial velocity measurements, made with the state-of-the-art HARPS spectrograph (Lovis et al. 2006), are below the $\mathrm{m} / \mathrm{s}$ level, the associated systematic errors dominate the error budget. In particular, the ThAr lamps used to calibrate the spectrograph exhibit drifts and aging effects that will ultimately limit the precision of measurements made using this calibration technique (Lovis 2006). Iodine cell calibrators have similar limitations (Butler 1996). To detect Earth-like planets around sun-like stars, the calibration source must be accurate at the $\mathrm{cm} / \mathrm{s}$ level and stable at the same level over many years. We have developed a new calibrator based on laser comb technology that, when locked to GPS, will be accurate to a part in $10^{12}$ and stable to the same degree over decades. The concept of a laser comb calibrator was first suggested by Murphy (2007). In this proceeding, we give a brief overview of the calibration technology and comment on its utility for radial velocity planet searches. A more technical account of the astro-comb appears in Li (2008). 


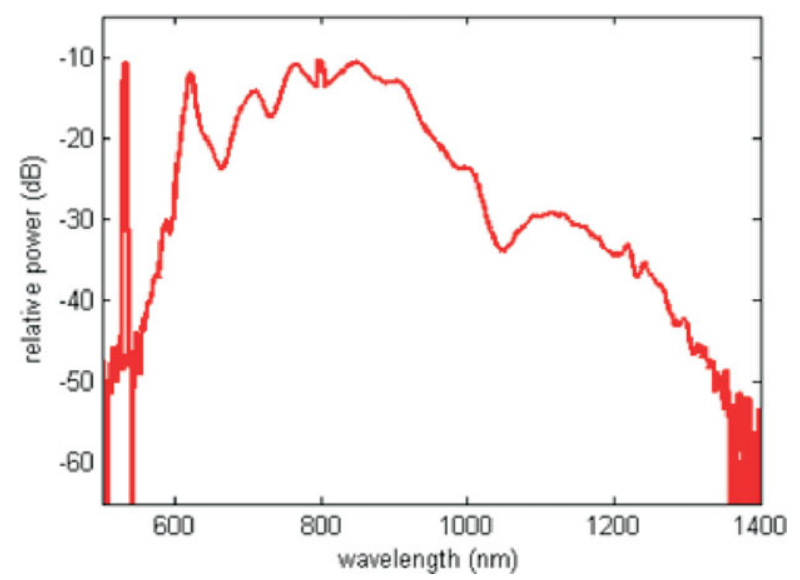

Figure 1. Spectrum of the comb light prior to filtering by the Fabry-Perot cavity. Individual comb lines are not resolved on this plot.

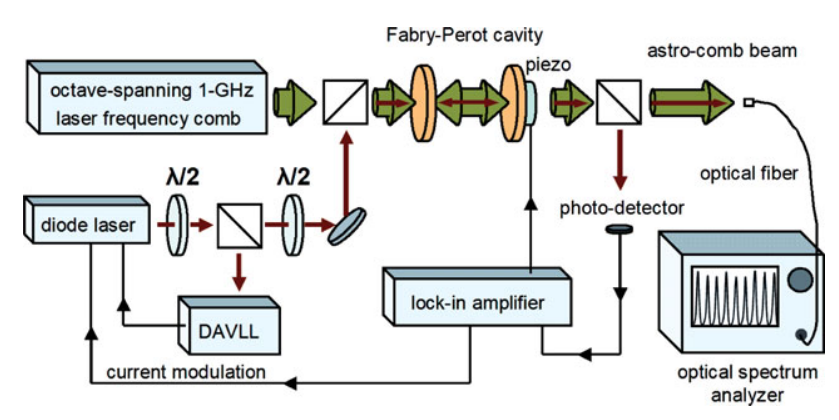

Figure 2. Schematic diagram of the astro-comb.

\section{Overview}

The astro-comb calibrator is a powerful combination of two laboratory instruments, optimized for use with astronomical spectrographs. The heart of the astro-comb is a femtosecond Titanuim:Sapphire laser that produces a comb of evenly spaced lines of nearly uniform intensity in the near infrared. The broad spectral envelope of comb light, shown in Fig. 1, is determined by the mirrors in the laser cavity. Individual comb lines are spaced by the comb laser's pulse repetition rate, in this case, $1 \mathrm{GHz}$ (for an introductory treatment of laser frequency combs, see Cundiff (2008) and Bergquist (2001)). While useful for frequency metrology and laboratory spectroscopy, a comb of such closely spaced lines will appear as a continuum to even the highest resolution astronomical spectrographs. We therefore place a Fabry-Perot (FP) filtering cavity at the source comb output, as shown in Fig. 2. The resulting spectrum consists of individual comb lines spaced by the FP cavity's free spectral range. By tuning the spacing between the mirrors of the FP cavity, we can select an effective comb line spacing of up to $40 \mathrm{GHz}$, and thus optimize the calibrator for use with instruments of different spectral resolving power.

The properties of the laser comb itself determine the precision and stability of the calibration lines. Both the repetition rate and overall frequency offset of the laser comb are locked to low-noise frequency synthesizers, which stabilizes the frequency of comb 
lines to a part in $10^{12}$ for times ranging from a few seconds to a several hours. The astrocomb will maintain this level of stability indefinitely when the frequency synthesizers are locked to Global Positioning System (GPS) clocks.

Accurate knowledge of the comb line frequencies is essential for calibrating individual radial velocity measurements as well as for comparing measurements made at different times. Referencing the GPS atomic clock also determines the absolute frequency of the comb lines to a part in $10^{12}$. The long-term stability of GPS will allow radial velocity measurements made at different times and using different instruments to be compared at the $\mathrm{cm} / \mathrm{s}$ level.

The FP cavity stability requirements are far less stringent than those for the comb. Because the width of the FP transmission peaks ( $150 \mathrm{MHz} F W H M)$ is large compared to the width of the comb lines $(\sim 1 \mathrm{kHz})$, a small amount of jitter in the FP will not adversely affect the calibration spectrum. We stabilize the FP cavity by locking it to a transition in atomic $\mathrm{Rb}$. With the positions of the individual FP transmission peaks stabilized to better than $500 \mathrm{kHz}$, we find that the amplitude of the comb lines changes by less than $0.1 \%$ and that the error in each calibration line position introduced by the changing suppression of neighboring comb lines is not more than $0.6 \mathrm{~cm} / \mathrm{s}$.

\section{Outlook}

We have built an astrocomb in the laboratory with a $40 \mathrm{GHz}$ line spacing and bandwidth of approximately $100 \mathrm{~nm}$ centered on $850 \mathrm{~nm}$. Each astro-comb calibration line has an accuracy and stability of a part in $10^{12}$. We plan to deploy this prototype comb on the Tillinghast Reflector Echelle Spectrograph (TRES) on Mt. Hopkins in July, 2008. Our initial tests of the astro-comb will have a dual purpose. First, we will demonstrate the utility of laser combs to calibrate high-resolution echelle spectrographs. Second, we will begin a program to characterize late-type $\mathrm{M}$ stars for exoplanet surveys. Late-type M-dwarfs are significantly brighter in the spectral range covered by the astro-comb than they are in the traditional spectral range used for radial velocity exoplanet searches. The TRES instrument was designed with M-stars in mind, but lacks a suitable calibrator at wavelengths longer than $800 \mathrm{~nm}$ because bright, broad, drifting Ar lines dominate the ThAr spectrum in that range (Lovis 2006). The prototype astro-comb will fill that void. Looking toward the future, we are exploring various techniques for expanding the comb's spectrum to range from 400-1000 nm, including frequency doubling and up-conversion in nonlinear crystals. While creating such a broad spectrum remains a technical challenge, we expect to have a functioning prototype for use with the HARPS-North spectrograph by early 2010 .

\section{References}

Bergquist, J. C., Jefferts, S. R., \& Wineland, D. J. 2001 Phys. Today 54, 37

Butler, R. P., Marcy, G. W., Williams, E., McCarthy, C., Dosanjh, P., \& Vogt, S. S. 1996 PASP, 108,500

Cundiff, S., Ye, J., \& Hall, J. 2008 Sci. Am. 298, 74

Li, C.-h., Benedick, A. J., Fendel, P., Glenday, A. G.; Kärtner, F. X.; Phillips, D. F.; Sasselov, D.; Szentgyorgyi, A., \& Walsworth, R. L. 2008, Nature, 452, 610

Lovis, C., Pepe, F., Bouchy, F., Lo Curto, G., Mayor, M., Pasquini, L., Queloz, D., Rupprecht, G., Udry, S., \& Zucker, S. 2006 Proc. SPIE 6269, 62690P23

Murphy, M. T., Udem, Th., Holzwarth, R., Sizmann, A., Pasquini, L., Araujo-Hauck, C., Dekker, H., D'Odorico, S., Fischer, M., Hänsch, T. W., et al. 2007 Mon. Not. R. Astron. Soc. 380, 839 